\title{
Removal of nitrate and phosphate in groundwater using a single reagent composed of calcium-organic complexes
}

\author{
JIYOUNG KANG AND SUNG-WOOK JEEN*
}

Department of Earth and Environmental Sciences, Jeonbuk

National University, Jeonju-si, Jeollabuk-do, Korea

(*correspondence: sjeen@jbnu.ac.kr)

Nitrate and phosphate in groundwater can be transported to surface water by means of base flow, potentially exacerbating outbreak of eutrophication. Therefore, treatment of these nutrients is required before they reach to surface water bodies. The purposes of this study were to develop a method to simultaneously remove nitrate and phosphate in groundwater and to identify removal mechanisms. In this study, calcium-organic (citrate and lactate) complexes were proposed as reagents to remove the two nutrients. Batch and column experiments were conducted to evaluate treatability of reactive mixtures. The results of batch experiments showed that the proposed calcium-organic complexes can remove nitrate and phosphate simultaneously. Nitrate was removed through denitrification by denitrifying bacteria which use citrate or lactate as carbon sources. Microbial analysis for the soil used for the experiments showed that denitrifying bacteria were $75.1 \%$ among the total soil bacteria in terms of class. The mechanisms for phosphate removal appeared to be adsorption and precipitation of a phosphate mineral (e.g., hydroxyapatite). There was no significant difference in the removal efficiencies between the two organic mixtures. Column experiments conducted under the flowing conditions also showed that the reagents can be effective to remove nitrate and phosphate under the conditions similar to natural groundwater environments. Nitrate and phosphate were removed less than 1 and 4 days of residence time, respectively, within the columns filled with local soil. This study suggests that nitrate and phosphate in groundwater can be simultaneously removed using calcium-organic complexes, suggesting a promising method to alleviate potential eutrophication in surface water. This work was supported by the National Research Foundation of Korea (NRF) grant funded by the Korea government (MSIT) (NRF2019R1A2C1086667). 